\title{
Cancer biomarkers for targeted therapy
}

\author{
Delong Liu ${ }^{1,2}$
}

\begin{abstract}
Tumor-associated antigens (TAA) or cancer biomarkers are major targets for cancer therapies. Antibody- based agents targeting the cancer biomarkers include monoclonal antibodies (MoAbs), radiolabeled MoAbs, bispecific $T$ cell engagers, and antibody-drug conjugates. Antibodies targeting CD19, CD20, CD22, CD30, CD33, CD38, CD79B and SLAMF7 are in clinical applications for hematological malignancies. CD123, CLL-1, B cell maturation antigen, and CD138 are targets for cancer immunotherapeutic agents, including the chimeric antigen receptor - engineered T cells. Immune checkpoint inhibitors (ICls) against PD-1, PD-L1, and CTLA-4 have led to the revolution of cancer immunotherapy. More ICls targeting IDO, LAG3, TIM-3, TIGIT, SIGLECS, VISTA and CD47 are being explored. Small molecule inhibitors (SMIs) against tyrosine kinase oncoproteins such as BCR-ABL, JAK2, Bruton tyrosine kinase, FLT3, EGFR, ALK, HER2, VEGFR, FGFR, MEK, and MET have fundamentally changed the landscape of cancer therapy. SMls against BCL-2, IDHs, BRAF, PI3 kinase, mTOR, PARP, and CDKs have become the mainstay in the treatment of a variety of cancer types. To reduce and avoid off-tumor toxicities, cancer-specific TAAs such as CD33 are being manufactured through systems biology approach. Search for novel biomarkers and new designs as well as delivery methods of targeted agents are fueling the next wave of advances in cancer therapy.
\end{abstract}

Keywords: Biomarker, Tumor-associated antigen, BiTE, Antibody-drug conjugate, CAR-T

Tumor-associated antigens (TAA) or cancer biomarkers are major targets for cancer therapies. Antibody- based agents targeting cancer biomarkers include monoclonal antibodies (MoAbs), radiolabeled MoAbs, bispecific $\mathrm{T}$ cell engagers (BiTEs), and antibody-drug conjugates (ADCs) [1-6]. In the past few years, chimeric antigen receptor- engineered $\mathrm{T}$ cells (CAR - $\mathrm{T}$ ) have become a major breakthrough in cancer immunotherapy [7-12]. In addition to the improvement in the design and manufacture of these targeted agents, search for new cancer biomarkers becomes equally critical. More agents targeting the following major biomarkers are rapidly migrating from bench to bedside for cancer therapy.

\section{CD19, the most targeted biomarker}

CD19 is by far the most targeted biomarker for cancer immunotherapy [13]. One BiTE (blinatumomab) and two CAR-T products (tisagenlecleucel and axicabtagene ciloleucel) have been approved for clinical applications $[2,9,14,15]$. More CD19 ADCs are in clinical trials, including coltuximab ravtansine (SAR3419), denintuzumab mafodotin (SGN-CD19A), loncastuximab tesirine (ADCT-402) [16-19]. It is worthwhile to note that CD19-targeted CAR-T, tisgenlecleucel, has shown activity against refractory /relapsed multiple myeloma in conjunction with high dose melphalan and autologous stem cell transplantation [20,21].

\section{CD20, CD22, CD30, CD79b as targets for lymphoid malignancies}

MoAbs against CD20 have been widely used for lymphoid malignancies $[22,23]$. ADCs are increasingly used as chemoimmunotherapy. Four new ADCs have been approved for the treatment of lymphoid malignancies: brentuximab vedotin targeting CD30, inotuzumab ozogamicin and moxetumomab pasudotox targeting CD22, and polatuzumab vedotin targeting CD79b [3, 24-28]. More biomarkers are being targeted with ADCs or CAR- T cells. These biomarkers include CD25, CD37, CD56, CD70, CD74, and CD138 [29].

\footnotetext{
Correspondence: Delong_liu@nymc.edu

${ }^{1}$ New York Medical College, Valhalla, NY 10595, USA

${ }^{2}$ The First Affiliated Hospital of Zhengzhou University, Zhengzhou 450052, China
} 


\section{CD33, CD123 and CLL-1 as targets for myeloid malignancies}

Gemtuzumab ozogamicin (GO) is an ADC against CD33 that is widely expressed on myeloid cells [30]. GO has been approved for newly diagnosed as well as refractory /relapsed (RR) acute myeloid leukemia (AML) [31]. GO may be used as a single agent or in combination with chemotherapy regimens [32-34]. In addition, several novel ADCs targeting CD33 are under clinical development. These include vadastuximab talirine (SGN-CD33A), IMGN779, and AVE9633 (huMy9-6-DM4) [35-37].

ADCs targeting CD123, such as IMGN632 and SGNCD123A, are being tested in clinical trials [38-41]. Further development of SGN-123A was however terminated due to safety concerns.

BiTE and ADCs targeting CLL-1 are currently undergoing preclinical or early clinical investigations for AML $[42,43]$. CLL-1 - targeted CAR- T cells are in clinical trials for AML therapy $[44,45]$.

\section{Immune checkpoints for targeted immunotherapy} Immune checkpoint inhibitors (ICIs) against PD-1, PD-L1 and CTLA-4 have led to a fundamental paradigm shift in cancer immunotherapy [46-50]. One particular difference of ICIs from conventional chemotherapy is that the ICIs target immune cells instead of cancer cells and aim to modulate tumor microenvironment, leading to restoration of suppressed cancer immunity [51, 52]. More biomarkers of immune checkpoints including IDO, LAG3, TIM-3, TIGIT, SIGLECs, VISTA and CD47 are fueling the development of targeted agents [51, 53-59].

\section{B cell maturation antigen (BCMA) -targeted multiple myeloma therapy}

BCMA is expressed in normal B cells, MM cells and malignant B cells [60-62]. Several CAR-T cell products targeting BCMA are in advanced clinical development for multiple myeloma (MM), including bb2121, LCAR-B38M, JCARH125, MCARH171, P-BCMA-101, CT053, and CT103A [63, 64]. In a recent report of a phase I study, 33 patients received bb2121 with an overall response rate (ORR) of 85\% [65]. Sixteen patients were negative for minimal residue disease (MRD). LCAR-B38M is also in late stage clinical development. This CAR-T product contains a CAR targeting two BCMA epitopes [66, 67]. In a recent report of the LEGEND-2 trial, 57 patients who received infusion of LCAR-B38M CAR-T cells had an ORR $=$ PR or better) of $88 \%$ [67]. In addition, BCMA is being targeted with BiTE and ADCs [68-71].

CS1 glycoprotein antigen (SLAMF7) is expressed on NK cells and MM cells. Elotuzumab is a MoAb that has been approved for RRMM therapy [72, 73]. CAR-T cells targeting SLAMF7 and light chains are in active development for therapy of RRMM [63, 64].

\section{Biomarkers for solid tumor immunotherapy}

CD133-targeted CAR T cells have been used for solid tumors including cholangiocarcinoma [74-76]. Mesothelin- targeted CAR-T cells have been reported in mesothelioma, lung cancer, breast cancer, gastric cancer and pancreatic cancer [77, 78]. EGFR, HER2, and claudin18.2 are favorite targets for solid tumor immunotherapy including CAR-T and MoAbs [79-82]. T cell receptorengineered $\mathrm{T}$ cells against AFP and MAGE-A1 have been reported for immunotherapy of solid tumors [83-88].

\section{Tyrosine kinase biomarkers as targets of small molecule inhibitors}

Started with the BCR-ABL tyrosine kinase inhibitor (TKI), imatinib [89, 90], TKIs have become the mainstay of targeted oral agents for many cancer types [91-94]. Inhibitors of BCR-ABL, JAK2, FLT3, Bruton tyrosine kinase have led to a paradigm shift in the management of leukemias [95-100]. TKIs targeting a variety of tyrosine kinase oncoproteins, such as EGFR, ALK, HER2, FGFR, VEGFR, RET, MET, MEK have markedly changed the therapeutic landscape of such cancers as non-small cell lung cancer, breast cancer, bladder cancer, liver cancer, and renal cell carcinoma [101-106].

\section{BCL-2, IDHs, BRAF, PI3 kinase, mTOR, PARP and CDKs as targets of small molecule inhibitors}

Biomarkers of many non-tyrosine kinase oncoproteins are major targets also for cancer therapy. Inhibitors of BCL-2, isocitrate dehydrogenases (IDH1 and IDH2), PI3 kinase, BRAF, mTOR, PARP and CDK have vastly expanded the armamentarium against a variety of cancer types, such as leukemia, lymphoma, melanoma, breast cancer, and ovarian cancer [107-116].

\section{Engineering biomarkers through systems biology for cancer therapy}

TAAs are frequently expressed in normal tissues, therefore are not truly cancer-specific. Great efforts are being focused on engineering TAAs to improve or confer better cancer-specificity. CD33 is a myeloid marker and the target of the GO ADC in AML. BiTE and CAR-T cells are also being studied for clinical therapy of AML $[117,118]$. However, off-tumor toxicities due to expression of CD33 in normal hematopoietic cells restrict the clinical applications. When CD33 gene was knocked out from the human hematopoietic stem and progenitor cells (HSPC), CD33- targeting CAR-T cells specifically eliminated AML cells without myelotoxicity in recipients transplanted with CD33- null HSPCs [119]. Clinically, the similar approach using systems biology 
to engineer HSPCs has been tested in a HIV+ patient with highly refractory acute lymphoblastic leukemia (ALL) [120]. In this case, CCR-5 of a fully HLAmatched allogeneic normal donor HSPCs were ablated with CRISPR technology. The donor HSPCs with ablated CCR-5 were transplanted into the HIV+ patient. The ALL went into complete remission with persistence of CCR-5 negative hematopoiesis. This approach using systems biology and engineering opens up a new era of manufactured cancer-specific biomarkers for targeted cancer therapy. Search for novel biomarkers and new designs as well as delivery methods such as nanotechnology of targeted agents are fueling the next wave of advances in cancer therapy.

\section{Abbreviation}

CAR: Chimeric antigen receptor

\section{Acknowledgements}

I wish to dedicate this editorial to my mentor, Prof. Zhao-you Tang, President Emeritus of the Shanghai Medical University, and to the 50th Anniversary of The Liver Cancer Institute of Zhongshan Hospital, Fudan University Shanghai School of Medicine, Shanghai, China. I am deeply indebted to Drs. Zihai Li and Chung-tsen Hsueh for their critical review of this manuscript.

\section{Author's contributions}

DL drafted and approved the final manuscript.

\section{Funding}

The study is partly supported by the First Affiliated Hospital of Zhengzhou University, Zhengzhou, China.

\section{Availability of data and materials}

This is not applicable.

\section{Ethics approval and consent to participate}

This is not applicable.

\section{Consent for publication}

This is not applicable.

\section{Competing interests}

The author declares that he has no competing interests.

\section{Published online: 15 November 2019}

\section{References}

1. Goede V, Fischer K, Busch R, Engelke A, Eichhorst B, Wendtner CM, Chagorova T, de la Serna J, Dilhuydy MS, IIImer T, Opat S, Owen CJ, Samoylova O, Kreuzer KA, Stilgenbauer S, Dohner H, Langerak AW, Ritgen M, Kneba M, Asikanius E, Humphrey K, Wenger M, Hallek M. Obinutuzumab plus chlorambucil in patients with CLL and coexisting conditions. N Engl J Med. 2014;370(12):1101-10

2. Kantarjian $H$, Stein A, Gokbuget N, Fielding AK, Schuh AC, Ribera JM, Wei A, Dombret H, Foa R, Bassan R, Arslan O, Sanz MA, Bergeron J, Demirkan F, Lech-Maranda E, Rambaldi A, Thomas X, Horst HA, Bruggemann M, Klapper W, Wood BL, Fleishman A, Nagorsen D, Holland C, Zimmerman Z, Topp MS. Blinatumomab versus chemotherapy for advanced acute lymphoblastic leukemia. N Engl J Med. 2017;376(9):836-47.

3. Kantarjian HM, DeAngelo DJ, Stelljes M, Martinelli G, Liedtke M, Stock W, Gokbuget N, O'Brien S, Wang K, Wang T, Paccagnella ML, Sleight B, Vandendries E, Advani AS. Inotuzumab Ozogamicin versus standard therapy for acute lymphoblastic leukemia. N Engl J Med. 2016;375(8):740-53.

4. Maury S, Chevret S, Thomas X, Heim D, Leguay T, Huguet F, Chevallier P, Hunault M, Boissel N, Escoffre-Barbe M, Hess U, Vey N, Pignon JM, Braun T, Marolleau JP, Cahn JY, Chalandon Y, Lheritier V, Beldjord K, Bene MC, Ifrah
$\mathrm{N}$, Dombret $\mathrm{H}$, for $\mathrm{G}$. Rituximab in B-lineage adult acute lymphoblastic leukemia. N Engl J Med. 2016;375(11):1044-53.

5. Verma S, Miles D, Gianni L, Krop IE, Welslau M, Baselga J, Pegram M, Oh D-Y, Diéras V, Guardino E, Fang L, Lu MW, Olsen S, Blackwell K. Trastuzumab Emtansine for HER2-positive advanced breast cancer. N Engl J Med. 2012; 367(19):1783-91.

6. Yu S, Li A, Liu Q, Yuan X, Xu H, Jiao D, Pestell RG, Han X, Wu K. Recent advances of bispecific antibodies in solid tumors. J Hematol Oncol. 2017; 10(1):155.

7. Zhang C, Liu J, Zhong JF, Zhang X. Engineering CAR-T cells. Biomarker Res. 2017;5(1):22.

8. Yu S, Li A, Liu Q, Li T, Yuan X, Han X, Wu K. Chimeric antigen receptor T cells: a novel therapy for solid tumors. J Hematol Oncol. 2017;10(1):78.

9. Maude SL, Laetsch TW, Buechner J, Rives S, Boyer M, Bittencourt H, Bader P, Verneris MR, Stefanski HE, Myers GD, Qayed M, De Moerloose B, Hiramatsu $\mathrm{H}$, Schlis K, Davis KL, Martin PL, Nemecek ER, Yanik GA, Peters C, Baruchel A, Boissel N, Mechinaud F, Balduzzi A, Krueger J, June CH, Levine BL, Wood P, Taran T, Leung M, Mueller KT, et al. Tisagenlecleucel in children and Young adults with B-cell lymphoblastic leukemia. N Engl J Med. 2018;378(5):439-48.

10. Porter $\mathrm{DL}$, Levine $\mathrm{BL}$, Kalos $\mathrm{M}$, Bagg A, June $\mathrm{CH}$. Chimeric antigen receptormodified T cells in chronic lymphoid leukemia. N Engl J Med. 2011;365(8): 725-33.

11. June $\mathrm{CH}, \mathrm{O}^{\prime}$ Connor RS, Kawalekar OU, Ghassemi S, Milone MC. CAR T cell immunotherapy for human cancer. Science. 2018;359(6382):1361-5.

12. Wei G, Ding L, Wang J, Hu Y, Huang H. Advances of CD19-directed chimeric antigen receptor-modified $T$ cells in refractory/relapsed acute lymphoblastic leukemia. Exp Hematol Oncol. 2017;6(1):10.

13. Liu B, Song Y, Liu D. Clinical trials of CAR-T cells in China. J Hematol Oncol. 2017;10(1):166.

14. Topp MS, Gökbuget N, Stein AS, Zugmaier G, O'Brien S, Bargou RC, Dombret H, Fielding AK, Heffner L, Larson RA, Neumann S, Foà R, Litzow M, Ribera J-M, Rambaldi A, Schiller G, Brüggemann M, Horst HA, Holland C, Jia C, Maniar T, Huber B, Nagorsen D, Forman SJ, Kantarjian HM. Safety and activity of blinatumomab for adult patients with relapsed or refractory Bprecursor acute lymphoblastic leukaemia: a multicentre, single-arm, phase 2 study. Lancet Oncol. 2015;16(1):57-66.

15. Neelapu SS, Locke FL, Bartlett NL, Lekakis LJ, Miklos DB, Jacobson CA, Braunschweig I, Oluwole OO, Siddiqi T, Lin Y, Timmerman JM, Stiff PJ, Friedberg JW, Flinn IW, Goy A, Hill BT, Smith MR, Deol A, Farooq U, McSweeney P, Munoz J, Avivi I, Castro JE, Westin JR, Chavez JC, Ghobadi A, Komanduri KV, Levy R, Jacobsen ED, Witzig TE, et al. Axicabtagene Ciloleucel CAR T-cell therapy in refractory large B-cell lymphoma. N Engl J Med. 2017; 377(26):2531-44

16. Caimi P, Kahl BS, Hamadani M, Carlo-Stella C, He S, Ungar D, Feingold J, Ardeshna KM, Radford J, Solh M, Heffner L, O'Connor OA. Safety and efficacy of Adct-402 (Loncastuximab Tesirine), a novel antibody drug conjugate, in relapsed/refractory follicular lymphoma and mantle cell lymphoma: interim results from the phase 1 first-in-human study. Blood. 2018;132:2874.

17. Coiffier B, Thieblemont C, de Guibert S, Dupuis J, Ribrag V, Bouabdallah R, Morschhauser F, Navarro R, Le Gouill S, Haioun C, Houot R, Casasnovas O, Holte H, Lamy T, Broussais F, Payrard S, Hatteville L, Tilly H. A phase II, single-arm, multicentre study of coltuximab ravtansine (SAR3419) and rituximab in patients with relapsed or refractory diffuse large B-cell lymphoma. Br J Haematol. 2016;173(5):722-30.

18. Fathi AT, Erba HP, Lancet JE, Stein EM, Ravandi F, Faderl S, Walter RB, Advani AS, DeAngelo DJ, Kovacsovics TJ, Jillella A, Bixby D, Levy MY, O'Meara MM, Ho PA, Voellinger J, Stein AS. A phase 1 trial of vadastuximab talirine combined with hypomethylating agents in patients with CD33-positive AML. Blood. 2018;132(11):1125-33.

19. Moskowitz $\mathrm{CH}$, Fanale MA, Shah BD, Advani RH, Chen R, Kim S, Kostic A, Liu TN, Peng J, Forero-Torres A. A phase 1 study of Denintuzumab Mafodotin (SGN-CD19A) in relapsed/Refactory B-lineage non-Hodgkin lymphoma. Blood. 2015;126(23):182

20. Garfall AL, Stadtmauer EA, Hwang WT, Lacey SF, Melenhorst JJ, Krevvata M, Carroll MP, Matsui WH, Wang Q, Dhodapkar MV, Dhodapkar K, Das R, Vogl DT, Weiss BM, Cohen AD, Mangan PA, Ayers EC, Nunez-Cruz S, Kulikovskaya I, Davis MM, Lamontagne A, Dengel K, Kerr ND, Young RM, Siegel DL, Levine BL, Milone MC, Maus MV, June CH. Anti-CD19 CAR T cells with highdose melphalan and autologous stem cell transplantation for refractory multiple myeloma. JCl Insight. 2018;3(8):1-14. 
21. Garfall AL, Maus MV, Hwang WT, Lacey SF, Mahnke YD, Melenhorst JJ, Zheng Z, Vogl DT, Cohen AD, Weiss BM, Dengel K, Kerr ND, Bagg A, Levine BL, June CH, Stadtmauer EA. Chimeric antigen receptor T cells against CD19 for multiple myeloma. N Engl J Med. 2015;373(11):1040-7.

22. Ribrag V, Koscielny S, Bosq J, Leguay T, Casasnovas O, Fornecker L-M, Recher C, Ghesquieres H, Morschhauser F, Girault S, Gouill SL, Ojeda-Uribe M, Mariette C, Cornillon J, Cartron G, Verge V, Chassagne-Clément C, Dombret H, Coiffier B, Lamy T, Tilly H, Salles G. Rituximab and dose-dense chemotherapy for adults with Burkitt's lymphoma: a randomised, controlled, open-label, phase 3 trial. Lancet. 2016;387(10036):2402-11.

23. Marcus R, Davies A, Ando K, Klapper W, Opat S, Owen C, Phillips E, Sangha $R$, Schlag R, Seymour JF, Townsend W, Trněný $M$, Wenger M, FingerleRowson G, Rufibach K, Moore T, Herold M, Hiddemann W. Obinutuzumab for the first-line treatment of follicular lymphoma. N Engl J Med. 2017; 377(14):1331-44.

24. Kreitman RJ, Tallman MS, Robak T, Coutre S, Wilson WH, Stetler-Stevenson M, FitzGerald DJ, Santiago L, Gao G, Lanasa MC, Pastan I. Minimal residual hairy cell leukemia eradication with moxetumomab pasudotox: phase 1 results and long-term follow-up. Blood. 2018;131(21):2331-4.

25. Morschhauser F, Flinn IW, Advani R, Sehn LH, Diefenbach C, Kolibaba K, Press OW, Salles G, Tilly H, Chen Al, Assouline S, Cheson BD, Dreyling M, Hagenbeek A, Zinzani PL, Jones S, Cheng J, Lu D, Penuel E, Hirata J, Wenger M, Chu YW, Sharman J. Polatuzumab vedotin or pinatuzumab vedotin plus rituximab in patients with relapsed or refractory non-Hodgkin lymphoma: final results from a phase 2 randomised study (ROMULUS). Lancet Haematol. 2019;6(5):e254-65.

26. Palanca-Wessels MC, Czuczman M, Salles G, Assouline S, Sehn LH, Flinn I, Patel MR, Sangha R, Hagenbeek A, Advani R, Tilly H, Casasnovas O, Press OW, Yalamanchili S, Kahn R, Dere RC, Lu D, Jones S, Jones C, Chu YW, Morschhauser F. Safety and activity of the anti-CD79B antibody-drug conjugate polatuzumab vedotin in relapsed or refractory B-cell nonHodgkin lymphoma and chronic lymphocytic leukaemia: a phase 1 study. Lancet Oncol. 2015;16(6):704-15.

27. Kreitman RJ, Dearden C, Zinzani PL, Delgado J, Karlin L, Robak T, Gladstone $D E$, le Coutre P, Dietrich S, Gotic M, Larratt L, Offner F, Schiller G, Swords R, Bacon L, Bocchia M, Bouabdallah K, Breems DA, Cortelezzi A, Dinner S, Doubek M, Gjertsen BT, Gobbi M, Hellmann A, Lepretre S, Maloisel F, Ravandi F, Rousselot P, Rummel M, Siddiqi T, et al. Moxetumomab pasudotox in relapsed/refractory hairy cell leukemia. Leukemia. 2018;32(8): 1768-77.

28. Younes A, Bartlett NL, Leonard JP, Kennedy DA, Lynch CM, Sievers EL, Forero-Torres A. Brentuximab Vedotin (SGN-35) for relapsed CD30-positive lymphomas. N Engl J Med. 2010;363(19):1812-21.

29. Yu B, Liu D. Antibody-drug conjugates in clinical trials for lymphoid malignancies and multiple myeloma. J Hematol Oncol. 2019;12(1):94.

30. Khan N, Hills RK, Virgo P, Couzens S, Clark N, Gilkes A, Richardson P, Knapper S, Grimwade D, Russell NH, Burnett AK, Freeman SD. Expression of CD33 is a predictive factor for effect of gemtuzumab ozogamicin at different doses in adult acute myeloid leukaemia. Leukemia. 2017;31(5):1059-68.

31. Appelbaum FR, Bernstein ID. Gemtuzumab ozogamicin for acute myeloid leukemia. Blood. 2017:130(22):2373-6.

32. Ashaye AO, Khankhel Z, Xu Y, Fahrbach K, Mokgokong R, Orme ME, Lang K, Cappelleri JC, Mamolo C. A comparative evaluation of gemtuzumab ozogamicin + daunorubicin-cytarabine and other treatments for newly diagnosed acute myeloid leukemia. Future Oncol. 2019;15(6):663-81.

33. Amadori S, Suciu S, Selleslag D, Aversa F, Gaidano G, Musso M, Annino $L$, Venditti A, Voso MT, Mazzone C, Magro D, De Fabritiis P, Muus P, Alimena G, Mancini M, Hagemeijer A, Paoloni F, Vignetti M, Fazi P, Meert L, Ramadan SM, Willemze R, de Witte T, Baron F. Gemtuzumab Ozogamicin Versus Best Supportive Care in Older Patients With Newly Diagnosed Acute Myeloid Leukemia Unsuitable for Intensive Chemotherapy: Results of the Randomized Phase III EORTC-GIMEMA AML-19 Trial. J Clin Oncol. 2016;34(9):972-9.

34. Amadori S, Suciu S, Stasi R, Salih HR, Selleslag D, Muus $P$, De Fabritiis $P$, Venditti A, Ho AD, Lubbert M, Thomas X, Latagliata R, Halkes CJ, Falzetti F, Magro D, Guimaraes JE, Berneman Z, Specchia G, Karrasch M, Fazi P, Vignetti M, Willemze R, de Witte T, Marie JP. Sequential combination of gemtuzumab ozogamicin and standard chemotherapy in older patients with newly diagnosed acute myeloid leukemia: results of a randomized phase III trial by the EORTC and GIMEMA consortium (AML-17). J Clin Oncol. 2013;31(35):4424-30.
35. Adams S, Kelly M, McCarthy R, Wilhelm A, Watkins K, Lanieri L, Sloss CM, Romanelli A. IMGN779, a next generation CD33-targeting ADC, combines effectively with Cytarabine in Acute Myeloid Leukemia (AML) preclinical models, resulting in increased DNA damage response, cell cycle arrest and apoptosis in vitro and prolonged survival in vivo. Blood. 2017;130(Suppl 1): 1357.

36. Kung Sutherland MS, Walter RB, Jeffrey SC, Burke PJ, Yu C, Kostner H, Stone I, Ryan MC, Sussman D, Lyon RP, Zeng W, Harrington KH, Klussman K, Westendorf L, Meyer D, Bernstein ID, Senter PD, Benjamin DR, Drachman JG, McEarchern JA. SGN-CD33A: a novel CD33-targeting antibody-drug conjugate using a pyrrolobenzodiazepine dimer is active in models of drugresistant AML. Blood. 2013;122(8):1455-63.

37. Lapusan S, Vidriales MB, Thomas $X$, de Botton S, Vekhoff A, Tang R, Dumontet C, Morariu-Zamfir R, Lambert JM, Ozoux ML, Poncelet P, San Miguel JF, Legrand O, DeAngelo DJ, Giles FJ, Marie JP. Phase I studies of AVE9633, an anti-CD33 antibody-maytansinoid conjugate, in adult patients with relapsed/refractory acute myeloid leukemia. Investig New Drugs. 2012; 30(3):1121-31

38. Li F, Sutherland MK, Yu C, Walter RB, Westendorf L, Valliere-Douglass J, Pan L, Cronkite A, Sussman D, Klussman K, Ulrich M, Anderson ME, Stone IJ, Zeng W, Jonas M, Lewis TS, Goswami M, Wang SA, Senter PD, Law CL, Feldman EJ, Benjamin DR. Characterization of SGN-CD123A, a potent CD123-directed antibody-drug conjugate for acute myeloid leukemia. Mol Cancer Ther. 2018;17(2):554-64.

39. Sutherland MSK, Yu CP, Walter RB, Westendorf L, Valliere-Douglass J, Pan $L$, Sussman D, Anderson M, Zeng WP, Stone I, Klussman K, Ulrich M, Jonas M, Senter P, Drachman JG, Benjamin D. SGN-CD123A, a Pyrrolobenzodiazepine dimer linked anti-CD123 antibody drug conjugate, demonstrates effective anti-leukemic activity in multiple preclinical models of AML. Blood. 2015; 126(23):330

40. Adams S, Wilhelm A, Harvey L, Bai C, Yoder N, Kovtun Y, Chittenden T, Pinkas J. IMGN632: A CD123-Targeting Antibody-Drug Conjugate (ADC) with a novel DNA-Alkylating payload, is highly active and prolongs survival in Acute Myeloid Leukemia (AML) xenograft models. Blood. 2016;128(22):2832.

41. Kovtun Y, Jones G, Audette C, Harvey L, Gerard B, Wilhelm A, Bai C, Adams S, Goldmacher VS, Chari R, Chittenden T. A CD123-targeting Antibody-Drug Conjugate (ADC), IMGN632, designed to eradicate Acute Myeloid Leukemia (AML) cells while sparing normal bone marrow cells. Blood. 2016;128(22): 768.

42. Beyar-Katz O, Gill S. Novel approaches to acute myeloid leukemia immunotherapy. Clin Cancer Res. 2018;24(22):5502.

43. Leong SR, Sukumaran S, Hristopoulos M, Totpal K, Stainton S, Lu E, Wong A, Tam L, Newman R, Vuillemenot BR, Ellerman D, Gu C, Mathieu M, Dennis MS, Nguyen A, Zheng B, Zhang C, Lee G, Chu YW, Prell RA, Lin K, Laing ST, Polson AG. An anti-CD3/anti-CLL-1 bispecific antibody for the treatment of acute myeloid leukemia. Blood. 2017;129(5):609-18.

44. Wang J, Chen S, Xiao W, Li W, Wang L, Yang S, Wang W, Xu L, Liao S, Liu W, Wang Y, Liu N, Zhang J, Xia X, Kang T, Chen G, Cai X, Yang H, Zhang X, Lu $Y$, Zhou P. CAR-T cells targeting CLL-1 as an approach to treat acute myeloid leukemia. J Hematol Oncol. 2018;11(1):7.

45. Tashiro H, Sauer T, Shum T, Parikh K, Mamonkin M, Omer B, Rouce RH, Lulla P, Rooney CM, Gottschalk S, Brenner MK. Treatment of acute myeloid leukemia with T cells expressing chimeric antigen receptors directed to Ctype lectin-like molecule 1. Mol Ther. 2017;25(9):2202-13.

46. Diggs LP, Hsueh EC. Utility of PD-L1 immunohistochemistry assays for predicting PD-1/PD-L1 inhibitor response. Biomarker Res. 2017;5(1):12.

47. Motzer RJ, Penkov K, Haanen J, Rini B, Albiges L, Campbell MT, Venugopal B, Kollmannsberger C, Negrier S, Uemura M, Lee JL, Vasiliev A, Miller WH, Gurney H, Schmidinger M, Larkin J, Atkins MB, Bedke J, Alekseev B, Wang J, Mariani M, Robbins PB, Chudnovsky A, Fowst C, Hariharan S, Huang B, di Pietro A, Choueiri TK. Avelumab plus Axitinib versus Sunitinib for advanced renal-cell carcinoma. N Engl J Med. 2019;380(12):1103-15.

48. Postow MA, Chesney J, Pavlick AC, Robert C, Grossmann K, McDermott D, Linette GP, Meyer N, Giguere JK, Agarwala SS, Shaheen M, Ernstoff MS, Minor D, Salama AK, Taylor M, Ott PA, Rollin LM, Horak C, Gagnier P, Wolchok JD, Hodi FS. Nivolumab and ipilimumab versus ipilimumab in untreated melanoma. N Engl J Med. 2015;372(21):2006-17.

49. Robert C, Long GV, Brady B, Dutriaux C, Maio M, Mortier L, Hassel JC, Rutkowski P, McNeil C, Kalinka-Warzocha E, Savage KJ, Hernberg MM, Lebbe C, Charles J, Mihalcioiu C, Chiarion-Sileni V, Mauch C, Cognetti F, Arance A, Schmidt H, Schadendorf D, Gogas H, Lundgren-Eriksson L, Horak C, Sharkey 
B, Waxman IM, Atkinson V, Ascierto PA. Nivolumab in previously untreated melanoma without BRAF mutation. N Engl J Med. 2015;372(4):320-30.

50. Robert C, Schachter J, Long GV, Arance A, Grob JJ, Mortier L, Daud A, Carlino MS, McNeil C, Lotem M, Larkin J, Lorigan P, Neyns B, Blank CU, Hamid O, Mateus C, Shapira-Frommer R, Kosh M, Zhou H, Ibrahim N, Ebbinghaus S, Ribas A, Investigators K. Pembrolizumab versus Ipilimumab in advanced melanoma. N Engl J Med. 2015;372(26):2521-32.

51. Wang J, Sun J, Liu LN, Flies DB, Nie X, Toki M, Zhang J, Song C, Zarr M, Zhou X, Han X, Archer KA, O'Neill T, Herbst RS, Boto AN, Sanmamed MF, Langermann S, Rimm DL, Chen L. Siglec-15 as an immune suppressor and potential target for normalization cancer immunotherapy. Nat Med. 2019; 25(4):656-66.

52. Wang J, Yuan R, Song W, Sun J, Liu D, Li Z. PD-1, PD-L1 (B7-H1) and tumorsite immune modulation therapy: the historical perspective. J Hematol Oncol. 2017;10(1):34.

53. Liu M, Wang X, Wang L, Ma X, Gong Z, Zhang S, Li Y. Targeting the IDO1 pathway in cancer: from bench to bedside. J Hematol Oncol. 2018;11(1):100.

54. Ok CY, Young KH. Checkpoint inhibitors in hematological malignancies. J Hematol Oncol. 2017:10(1):103.

55. Crocker PR, Paulson JC, Varki A. Siglecs and their roles in the immune system. Nat Rev Immunol. 2007;7(4):255-66.

56. Gholamin S, Mitra SS, Feroze AH, Liu J, Kahn SA, Zhang M, Esparza R, Richard C, Ramaswamy V, Remke M, Volkmer AK, Willingham S, Ponnuswami A, McCarty A, Lovelace P, Storm TA, Schubert S, Hutter G, Narayanan C, Chu P, Raabe EH, Harsh G, Taylor MD, Monje M, Cho Y-J, Majeti R, Volkmer JP, Fisher PG, Grant G, Steinberg GK, et al. Disrupting the CD47-SIRPa anti-phagocytic axis by a humanized anti-CD47 antibody is an efficacious treatment for malignant pediatric brain tumors. Sci Transl Med. 2017;9(381):eaaf2968.

57. Shang L, Buatois V, Hatterer E, Chauchet X, Haddouk H, Majocchi S, Masternak K, Kosco-Vilbois MH, Fischer N, Ferlin WG. Abstract 546: selectively targeting CD47 with bispecific antibody to efficiently eliminate mesothelin-positive solid tumors. Cancer Res. 2019;79(13 Supplement):546.

58. Manieri NA, Chiang EY, Grogan JL. TIGIT: a key inhibitor of the cancer immunity cycle. Trends Immunol. 2017;38(1):20-8.

59. Lines JL, Pantazi E, Mak J, Sempere LF, Wang L, O'Connell S, Ceeraz S, Suriawinata AA, Yan S, Ernstoff MS, Noelle R. VISTA is an immune checkpoint molecule for human T cells. Cancer Res. 2014;74(7):1924-32.

60. Sanchez E, Li M, Kitto A, Li J, Wang CS, Kirk DT, Yellin O, Nichols CM, Dreyer MP, Ahles CP, Robinson A, Madden E, Waterman GN, Swift RA, Bonavida B, Boccia R, Vescio RA, Crowley J, Chen H, Berenson JR. Serum B-cell maturation antigen is elevated in multiple myeloma and correlates with disease status and survival. Br J Haematol. 2012;158(6):727-38.

61. Xu S, Lam KP. B-cell maturation protein, which binds the tumor necrosis factor family members BAFF and APRIL, is dispensable for humoral immune responses. Mol Cell Biol. 2001;21(12):4067-74.

62. Shu $H B$, Johnson $H$. B cell maturation protein is a receptor for the tumor necrosis factor family member TALL-1. Proc Natl Acad Sci U S A. 2000; 97(16):9156-61.

63. Killock D. Anti-BCMA CAR T cells for MM. Nat Rev Clin Oncol. 2019;16(8):465.

64. Timmers M, Roex G, Wang Y, Campillo-Davo D, Van Tendeloo VFI, Chu Y, Berneman ZN, Luo F, Van Acker HH, Anguille S. Chimeric Antigen Receptor-Modified T Cell Therapy in Multiple Myeloma: Beyond B Cell Maturation Antigen. Front Immunol. 2019;10(1613). https://doi.org/10. 3389/fimmu.2019.01613.

65. Raje N, Berdeja J, Lin Y, Siegel D, Jagannath S, Madduri D, Liedtke M, Rosenblatt J, Maus MV, Turka A, Lam L-P, Morgan RA, Friedman K, Massaro M Wang J, Russotti G, Yang Z, Campbell T, Hege K, Petrocca F, Quigley MT, Munshi N, Kochenderfer JN. Anti-BCMA CAR T-cell therapy bb2121 in relapsed or refractory multiple myeloma. N Engl J Med. 2019;380(18):1726-37.

66. Xu J, Chen LJ, Yang SS, Sun Y, Wu W, Liu YF, Xu J, Zhuang Y, Zhang W, Weng $X Q$, Wu J, Wang $Y$, Wang J, Yan H, Xu WB, Jiang H, Du J, Ding XY, Li B, Li JM, Fu WJ, Zhu J, Zhu L, Chen Z, Fan XF, Hou J, Li JY, Mi JQ, Chen SJ. Exploratory trial of a biepitopic CAR T-targeting B cell maturation antigen in relapsed/refractory multiple myeloma. Proc Natl Acad Sci U S A. 2019; 116(19):9543-51.

67. Zhao W-H, Liu J, Wang B-Y, Chen Y-X, Cao X-M, Yang Y, Zhang Y-L, Wang F$X$, Zhang P-Y, Lei B, Gu L-F, Wang J-L, Yang N, Zhang R, Zhang $H$, Shen $Y$, Bai J, Xu Y, Wang X-G, Zhang R-L, Wei L-L, Li Z-F, Li Z-Z, Geng Y, He Q, Zhuang Q-C, Fan X-H, He A-L, Zhang W-G. A phase 1, open-label study of LCAR-B38M, a chimeric antigen receptor T cell therapy directed against B cell maturation antigen, in patients with relapsed or refractory multiple myeloma. J Hematol Oncol. 2018;11(1):141.

68. Swan D, Lynch K, Gurney M, O'Dwyer M. Current and emerging immunotherapeutic approaches to the treatment of multiple myeloma. In: Therapeutic advances in hematology, vol. 10; 2019.

69. Eckhert E, Hewitt R, Liedtke M. B-cell maturation antigen directed monoclonal antibody therapies for multiple myeloma. Immunotherapy. 2019;11(9):801-11.

70. Cho SF, Anderson KC, Tai YT. Targeting B Cell Maturation Antigen (BCMA) in multiple myeloma: potential uses of BCMA-based immunotherapy. Front Immunol. 2018;9:1821.

71. Tai YT, Mayes PA, Acharya C, Zhong MY, Cea M, Cagnetta A, Craigen J, Yates J, Gliddon L, Fieles W, Hoang B, Tunstead J, Christie AL, Kung AL, Richardson $P$, Munshi NC, Anderson KC. Novel anti-B-cell maturation antigen antibodydrug conjugate (GSK2857916) selectively induces killing of multiple myeloma. Blood. 2014;123(20):3128-38.

72. Dimopoulos MA, Dytfeld D, Grosicki S, Moreau P, Takezako N, Hori M, Leleu X, LeBlanc R, Suzuki K, Raab MS, Richardson PG, Popa McKiver M, Jou Y-M, Shelat SG, Robbins M, Rafferty B, San-Miguel J. Elotuzumab plus Pomalidomide and dexamethasone for multiple myeloma. N Engl J Med. 2018;379(19):1811-22.

73. Lonial S, Dimopoulos M, Palumbo A, White D, Grosicki S, Spicka I, WalterCroneck A, Moreau P, Mateos M-V, Magen H, Belch A, Reece D, Beksac M, Spencer A, Oakervee H, Orlowski RZ, Taniwaki M, Röllig C, Einsele H, Wu KL, Singhal A, San-Miguel J, Matsumoto M, Katz J, Bleickardt E, Poulart V, Anderson KC, Richardson P. Elotuzumab therapy for relapsed or refractory multiple myeloma. N Engl J Med. 2015;373(7):621-31.

74. Glumac PM, LeBeau AM. The role of CD133 in cancer: a concise review. Clin Transl Med. 2018;7(1):18.

75. Barzegar Behrooz A, Syahir A, Ahmad S. CD133: beyond a cancer stem cell biomarker. J Drug Target. 2019;27(3):257-69.

76. Wang Y, Chen M, Wu Z, Tong C, Dai H, Guo Y, Liu Y, Huang J, Lv H, Luo C Feng K-C, Yang Q-M, Li X-L, Han W. CD133-directed CAR T cells for advanced metastasis malignancies: a phase I trial. Oncoimmunology. 2018; 7(7):e1440169.

77. Hassan R, Ho M. Mesothelin targeted cancer immunotherapy. Eur J Cancer (Oxford, England : 1990). 2008;44(1):46-53.

78. Kelly RJ, Sharon E, Pastan I, Hassan R. Mesothelin-targeted agents in clinical trials and in preclinical development. Mol Cancer Ther. 2012;11(3):517-25.

79. Wing A, Fajardo CA, Posey AD Jr, Shaw C, Da T, Young RM, Alemany R, June $\mathrm{CH}$, Guedan S. Improving CART-cell therapy of solid tumors with oncolytic virus-driven production of a bispecific T-cell engager. Cancer Immunol Res. 2018;6(5):605-16.

80. Guo Y, Wang Y, Han W. Chimeric antigen receptor-modified T cells for solid tumors: challenges and prospects. J Immunol Res. 2016;2016:3850839.

81. Yeku O, Li X, Brentjens RJ. Adoptive T-cell therapy for solid tumors. Am Soc Clin Oncol Educ Book. 2017:37:193-204.

82. Zhang $\mathrm{E}_{\mathrm{i}} \mathrm{Gu} \mathrm{J}, \mathrm{Xu} \mathrm{H}$. Prospects for chimeric antigen receptor-modified T cell therapy for solid tumors. Mol Cancer. 2018;17(1):7-7.

83. Xia Y, Tian X, Wang J, Qiao D, Liu X, Xiao L, Liang W, Ban D, Chu J, Yu J, Wang $R$, Tian G, Wang M. Treatment of metastatic non-small cell lung cancer with NY-ESO-1 specific TCR engineered-T cells in a phase I clinical trial: a case report. Oncol Lett. 2018;16(6):6998-7007.

84. Mo Z, Du P, Wang G, Wang Y. The multi-purpose tool of tumor immunotherapy: gene-engineered T cells. J Cancer. 2017:8(9):1690-703.

85. Zhang J, Wang L. The emerging world of TCR-T cell trials against Cancer: a systematic review. Technol Cancer Res Treat. 2019;18:1533033819831068.

86. Zhang Y, Li Y. T cell receptor-engineered T cells for leukemia immunotherapy. Cancer Cell Int. 2019;19:2-2.

87. Liu Y, Butterfield LH, Fu X, Song Z, Zhang X, Lu C, Ding G, Wu M. Lentivirally engineered dendritic cells activate AFP-specific T cells which inhibit hepatocellular carcinoma growth in vitro and in vivo. Int J Oncol. 2011;39(1):245-53.

88. Evdokimova VN, LiU Y, Potter DM, Butterfield LH. AFP-specific CD4+ helper T-cell responses in healthy donors and HCC patients. J Immunother (Hagerstown, Md : 1997). 2007;30(4):425-37.

89. Druker BJ, Sawyers CL, Kantarjian H, Resta DJ, Reese SF, Ford JM, Capdeville R, Talpaz M. Activity of a specific inhibitor of the BCR-ABL tyrosine kinase in the blast crisis of chronic myeloid leukemia and acute lymphoblastic leukemia with the Philadelphia chromosome. N Engl J Med. 2001;344(14):1038-42.

90. Druker BJ, Talpaz M, Resta DJ, Peng B, Buchdunger E, Ford JM, Lydon NB, Kantarjian H, Capdeville R, Ohno-Jones S, Sawyers CL. Efficacy and safety of 
a specific inhibitor of the BCR-ABL tyrosine kinase in chronic myeloid leukemia. N Engl J Med. 2001;344(14):1031-7.

91. Rossari F, Minutolo F, Orciuolo E. Past, present, and future of Bcr-Abl inhibitors: from chemical development to clinical efficacy. J Hematol Oncol. 2018;11(1):84

92. Ling $Y$, Xie $Q$, Zhang Z, Zhang $H$. Protein kinase inhibitors for acute leukemia. Biomarker Res. 2018;6(1):8.

93. Cortes JE, Kantarjian H, Shah NP, Bixby D, Mauro MJ, Flinn I, O'Hare T, Hu S, Narasimhan NI, Rivera VM, Clackson T, Turner CD, Haluska FG, Druker BJ, Deininger MWN, Talpaz M. Ponatinib in refractory Philadelphia chromosome-positive Leukemias. N Engl J Med. 2012;367(22):2075-88.

94. Goldman JM, Melo JV. Targeting the BCR-ABL tyrosine kinase in chronic myeloid leukemia. N Engl J Med. 2001;344(14):1084-6.

95. Byrd JC, Furman RR, Coutre SE, Flinn IW, Burger JA, Blum KA, Grant B, Sharman JP, Coleman M, Wierda WG, Jones JA, Zhao W, Heerema NA, Johnson AJ, Sukbuntherng J, Chang BY, Clow F, Hedrick E, Buggy JJ, James DF, O'Brien S. Targeting BTK with ibrutinib in relapsed chronic lymphocytic leukemia. N Engl J Med. 2013;369(1):32-42.

96. Byrd JC, Harrington B, O'Brien S, Jones JA, Schuh A, Devereux S, Chaves J Wierda WG, Awan FT, Brown JR, Hillmen P, Stephens DM, Ghia P, Barrientos JC, Pagel JM, Woyach J, Johnson D, Huang J, Wang X, Kaptein A, Lannutti BJ, Covey T, Fardis M, McGreivy J, Hamdy A, Rothbaum W, Izumi R, Diacovo TG, Johnson AJ, Furman RR. Acalabrutinib (ACP-196) in relapsed chronic lymphocytic leukemia. N Engl J Med. 2016;374(4):323-32.

97. Wang ML, Rule S, Martin P, Goy A, Auer R, Kahl BS, Jurczak W, Advani RH, Romaguera JE, Williams ME, Barrientos JC, Chmielowska E, Radford J, Stilgenbauer S, Dreyling M, Jedrzejczak WW, Johnson P, Spurgeon SE, Li L, Zhang L, Newberry K, Ou Z, Cheng N, Fang B, McGreivy J, Clow F, Buggy JJ, Chang BY, Beaupre DM, Kunkel LA, et al. Targeting BTK with ibrutinib in relapsed or refractory mantle-cell lymphoma. N Engl J Med. 2013;369(6): 507-16.

98. Grinfeld J, Nangalia J, Baxter EJ, Wedge DC, Angelopoulos N, Cantrill R, Godfrey AL, Papaemmanuil E, Gundem G, MacLean C, Cook J, O'Neil L, O'Meara S, Teague JW, Butler AP, Massie CE, Williams N, Nice FL, Andersen CL, Hasselbalch HC, Guglielmelli P, McMullin MF, Vannucchi AM, Harrison CN, Gerstung M, Green AR, Campbell PJ. Classification and personalized prognosis in myeloproliferative neoplasms. N Engl J Med. 2018;379(15): 1416-30.

99. Perl AE, Martinelli G, Cortes JE, Neubauer A, Berman E, Paolini S, Montesinos P, Baer MR, Larson RA, Ustun C, Fabbiano F, Erba HP, Di Stasi A, Stuart R, Olin R, Kasner M, Ciceri F, Chou W-C, Podoltsev N, Recher C, Yokoyama H, Hosono N, Yoon S-S, Lee J-H, Pardee T, Fathi AT, Liu C, Hasabou N, Liu X,

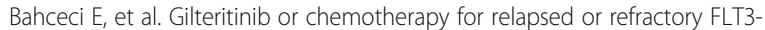
mutated AML. N Engl J Med. 2019;381(18):1728-40.

100. Stone RM, Mandrekar SJ, Sanford BL, Laumann K, Geyer S, Bloomfield CD, Thiede C, Prior TW, Döhner K, Marcucci G, Lo-Coco F, Klisovic RB, Wei A, Sierra J, Sanz MA, Brandwein JM, de Witte T, Niederwieser D, Appelbaum FR, Medeiros BC, Tallman MS, Krauter J, Schlenk RF, Ganser A, Serve H, Ehninger G, Amadori S, Larson RA, Döhner H. Midostaurin plus chemotherapy for acute myeloid leukemia with a FLT3 mutation. N Engl J Med. 2017;377(5): 454-64.

101. Su S, Wu Y-L. Clinical trials of tyrosine kinase inhibitors for lung cancer in China: a review. J Hematol Oncol. 2017;10(1):147

102. Soria J-C, Ohe Y, Vansteenkiste J, Reungwetwattana T, Chewaskulyong B, Lee KH, Dechaphunkul A, Imamura F, Nogami N, Kurata T, Okamoto I, Zhou C, Cho BC, Cheng Y, Cho EK, Voon PJ, Planchard D, Su W-C, Gray JE, Lee SM, Hodge R, Marotti M, Rukazenkov $Y$, Ramalingam SS. Osimertinib in untreated EGFR-mutated advanced non-small-cell lung Cancer. N Engl J Med. 2017;378(2):113-25.

103. Loriot $Y$, Necchi A, Park SH, Garcia-Donas J, Huddart R, Burgess E, Fleming M, Rezazadeh A, Mellado B, Varlamov S, Joshi M, Duran I, Tagawa ST, Zakharia Y, Zhong B, Stuyckens K, Santiago-Walker A, De Porre P, O'Hagan A, Avadhani A, Siefker-Radtke AO. Erdafitinib in locally advanced or metastatic urothelial carcinoma. N Engl J Med. 2019:381(4):338-48.

104. Long GV, Stroyakovskiy D, Gogas H, Levchenko E, de Braud F, Larkin J, Garbe C, Jouary T, Hauschild A, Grob JJ, Chiarion Sileni V, Lebbe C, Mandalà M, Millward M, Arance A, Bondarenko I, Haanen JBAG, Hansson J, Utikal J, Ferraresi V, Kovalenko N, Mohr P, Probachai V, Schadendorf D, Nathan P, Robert C, Ribas A, DeMarini DJ, Irani JG, Casey M, et al. Combined BRAF and MEK inhibition versus BRAF inhibition alone in melanoma. N Engl J Med. 2014;371(20):1877-88.
105. Choueiri TK, Escudier B, Powles T, Mainwaring PN, Rini BI, Donskov F, Hammers H, Hutson TE, Lee J-L, Peltola K, Roth BJ, Bjarnason GA, Géczi L, Keam B, Maroto P, Heng DYC, Schmidinger M, Kantoff PW, Borgman-Hagey A, Hessel C, Scheffold C, Schwab GM, Tannir NM, Motzer RJ. Cabozantinib versus Everolimus in advanced renal-cell carcinoma. N Engl J Med. 2015; 373(19):1814-23.

106. Schlumberger M, Tahara M, Wirth LJ, Robinson B, Brose MS, Elisei R, Habra MA, Newbold K, Shah MH, Hoff AO, Gianoukakis AG, Kiyota N, Taylor MH, Kim S-B, Krzyzanowska MK, Dutcus CE, de las Heras B, Zhu J, Sherman SI. Lenvatinib versus placebo in radioiodine-refractory thyroid Cancer. N Engl J Med. 2015;372(7):621-30.

107. Kopetz S, Grothey A, Yaeger R, Van Cutsem E, Desai J, Yoshino T, Wasan H, Ciardiello F, Loupakis F, Hong YS, Steeghs N, Guren TK, Arkenau H-T, GarciaAlfonso P, Pfeiffer P, Orlov S, Lonardi S, Elez E, Kim T-W, Schellens JHM, Guo C, Krishnan A, Dekervel J, Morris V, Calvo Ferrandiz A, Tarpgaard LS, Braun M, Gollerkeri A, Keir C, Maharry K, et al. Encorafenib, Binimetinib, and Cetuximab in BRAF V600E-mutated colorectal Cancer. N Engl J Med. 2019; 381(17):1632-43.

108. Jain N, Keating M, Thompson P, Ferrajoli A, Burger J, Borthakur G, Takahashi K, Estrov Z, Fowler N, Kadia T, Konopleva M, Alvarado Y, Yilmaz M, DiNardo C, Bose P, Ohanian M, Pemmaraju N, Jabbour E, Sasaki K, Kanagal-Shamanna R, Patel K, Jorgensen J, Garg N, Wang X, Sondermann K, Cruz N, Wei C, Ayala A, Plunkett W, Kantarjian $\mathrm{H}$, et al. Ibrutinib and Venetoclax for first-line treatment of CLL. N Engl J Med. 2019;380(22):2095-103.

109. Coleman RL, Fleming GF, Brady MF, Swisher EM, Steffensen KD, Friedlander M, Okamoto A, Moore KN, Efrat Ben-Baruch N, Werner TL, Cloven NG, Oaknin A, DiSilvestro PA, Morgan MA, Nam J-H, Leath CA, Nicum S, Hagemann AR, Littell RD, Cella D, Baron-Hay S, Garcia-Donas J, Mizuno M, Bell-McGuinn K, Sullivan DM, Bach BA, Bhattacharya S, Ratajczak CK, Ansell PJ, Dinh $\mathrm{MH}$, et al. Veliparib with first-line chemotherapy and as maintenance therapy in ovarian cancer. N Engl J Med. 2019;381. https://doi. org/10.1056/NEJMoa1909707.

110. Flaherty KT, Puzanov I, Kim KB, Ribas A, McArthur GA, Sosman JA, O'Dwyer PJ, Lee RJ, Grippo JF, Nolop K, Chapman PB. Inhibition of mutated, activated BRAF in metastatic melanoma. N Engl J Med. 2010;363(9):809-19.

111. González-Martín A, Pothuri B, Vergote I, DePont Christensen R, Graybill W, Mirza MR, McCormick C, Lorusso D, Hoskins P, Freyer G, Baumann K, Jardon K, Redondo A, Moore RG, Vulsteke C, O'Cearbhaill RE, Lund B, Backes F, Barretina-Ginesta P, Haggerty AF, Rubio-Pérez MJ, Shahin MS, Mangili G, Bradley WH, Bruchim I, Sun K, Malinowska IA, Li Y, Gupta D, Monk BJ. Niraparib in patients with newly diagnosed advanced ovarian cancer. New England J Med. 2019;381. https://doi.org/10.1056/NEJMoa1910962.

112. Hortobagyi GN, Stemmer SM, Burris HA, Yap Y-S, Sonke GS, Paluch-Shimon S, Campone M, Blackwell KL, André F, Winer EP, Janni W, Verma S, Conte P, Arteaga CL, Cameron DA, Petrakova K, Hart LL, Villanueva C, Chan A, Jakobsen E, Nusch A, Burdaeva O, Grischke E-M, Alba E, Wist E, Marschner N, Favret AM, Yardley D, Bachelot T, Tseng L-M, et al. Ribociclib as first-line therapy for HR-positive, advanced breast cancer. N Engl J Med. 2016;375(18) 1738-48.

113. Turner NC, Ro J, André F, Loi S, Verma S, Iwata H, Harbeck N, Loibl S, Huang Bartlett C, Zhang K, Giorgetti C, Randolph S, Koehler M, Cristofanilli M. Palbociclib in hormone-receptor-positive advanced breast cancer. N Engl J Med. 2015;373(3):209-19.

114. Liu D, Mamorska-Dyga A. Syk inhibitors in clinical development for hematological malignancies. J Hematol Oncol. 2017;10(1):145.

115. Im S-A, Lu Y-S, Bardia A, Harbeck N, Colleoni M, Franke F, Chow L, Sohn J, Lee K-S, Campos-Gomez S, Villanueva-Vazquez R, Jung K-H, Chakravartty A, Hughes G, Gounaris I, Rodriguez-Lorenc K, Taran T, Hurvitz S, Tripathy D. Overall survival with Ribociclib plus endocrine therapy in breast cancer. $\mathrm{N}$ Engl J Med. 2019;381(4):307-16.

116. Saygin C, Carraway HE. Emerging therapies for acute myeloid leukemia. J Hematol Oncol. 2017;10(1):93.

117. Hoseini SS, Guo H, Wu Z, Hatano MN, Cheung N-KV. A potent tetravalent Tcell-engaging bispecific antibody against CD33 in acute myeloid leukemia. Blood Adv. 2018;2(11):1250-8.

118. O'Hear C, Heiber JF, Schubert I, Fey G, Geiger TL. Anti-CD33 chimeric antigen receptor targeting of acute myeloid leukemia. Haematologica. 2015; 100(3):336-44.

119. Kim MY, Yu K-R, Kenderian SS, Ruella M, Chen S, Shin T-H, Aljanahi AA, Schreeder D, Klichinsky M, Shestova O, Kozlowski MS, Cummins KD, Shan X, Shestov M, Bagg A, Morrissette JJD, Sekhri P, Lazzarotto CR, Calvo KR, Kuhns 
DB, Donahue RE, Behbehani GK, Tsai SQ, Dunbar CE, Gill S. Genetic Inactivation of CD33 in Hematopoietic Stem Cells to Enable CAR T Cell Immunotherapy for Acute Myeloid Leukemia. Cell. 2018;173(6):1439-1453. e1419.

120. Xu L, Wang J, Liu Y, Xie L, Su B, Mou D, Wang L, Liu T, Wang X, Zhang B, Zhao L, Hu L, Ning H, Zhang Y, Deng K, Liu L, Lu X, Zhang T, Xu J, Li C, Wu $H$, Deng $H$, Chen H. CRISPR-edited stem cells in a patient with HIV and acute lymphocytic leukemia. N Engl J Med. 2019;381(13):1240-7.

\section{Publisher's Note}

Springer Nature remains neutral with regard to jurisdictional claims in published maps and institutional affiliations.

Ready to submit your research? Choose BMC and benefit from:

- fast, convenient online submission

- thorough peer review by experienced researchers in your field

- rapid publication on acceptance

- support for research data, including large and complex data types

- gold Open Access which fosters wider collaboration and increased citations

- maximum visibility for your research: over $100 \mathrm{M}$ website views per year

At $B M C$, research is always in progress.

Learn more biomedcentral.com/submissions 